\title{
Environmental factors associated with childhood norovirus diarrhoea in León, Nicaragua
}

\author{
S. BECKER-DREPS ${ }^{*} \dagger$, C. C. CUTHBERTSON ${ }^{2} \dagger$, F. BUCARDO ${ }^{3}$, J. VINJE $^{4}$, \\ M. PANIAGUA ${ }^{3}$, S. GIEBULTOWICZ ${ }^{5}$, F. ESPINOZA ${ }^{3}$ AND M. EMCH ${ }^{6}$ \\ ${ }^{1}$ Department of Family Medicine, University of North Carolina School of Medicine, Chapel Hill, North Carolina, \\ USA \\ ${ }^{2}$ Department of Epidemiology, UNC Gillings School of Global Public Health, Chapel Hill, North Carolina, USA \\ ${ }^{3}$ Department of Microbiology and Parasitology, Faculty of Medical Sciences, Universidad Nacional Autónoma de \\ Nicaragua, León, Nicaragua \\ ${ }^{4}$ Division of Viral Diseases, National Center for Immunization and Respiratory Diseases, Centers for Disease \\ Control and Prevention, Atlanta, Georgia, USA \\ ${ }^{5}$ Department of Family Medicine, School of Medicine, Oregon Health and Sciences University, Portland, OR, \\ USA \\ ${ }^{6}$ Department of Geography, University of North Carolina at Chapel Hill, North Carolina, USA
}

Received 20 July 2016; Final revision 17 January 2017; Accepted 25 January 2017; first published online 28 February 2017

\section{SUMMARY}

Norovirus is detected in one in five diarrhoea episodes in children, yet little is known about environmental risk factors associated with this disease, especially in low-income settings. The objective of this study was to examine environmental risk factors, and spatial and seasonal patterns of norovirus diarrhoea episodes in children in León, Nicaragua. We followed a population-based cohort of children under age 5 years for norovirus diarrhoea over a 1-year period. At baseline, characteristics of each household were recorded. Households were geocoded and spatial locations of garbage dumps, rivers, and markets were collected. In bivariate analysis we observed younger children and those with animals in their households were more likely to have experienced norovirus episodes. In adjusted models, younger children remained at higher risk for norovirus episodes, but only modest associations were observed with family and environmental characteristics. We next identified symptomatic children living in the same household and within $500 \mathrm{~m}$ buffer zones around the household of another child infected with the same genotype. Norovirus diarrhoea episodes peaked early in the rainy season. These findings contribute to our understanding of environmental factors and norovirus infection.

Key words: Norovirus, childhood diarrhoea, gastroenteritis, risk factors, Nicaragua.

\section{INTRODUCTION}

Diarrhoeal disease contributes substantially to childhood malnutrition and mortality. Globally, each

\footnotetext{
* Author for correspondence: S. Becker-Dreps, Department of Family Medicine, University of North Carolina School of Medicine, 590 Manning Drive, Chapel Hill, NC 27599-7595, USA. (Email: sbd@unc.edu)

$\dagger$ These authors contributed equally to this study.
}

year there are an estimated 1.7 billion episodes of diarrhoea [1] and one in five is associated with norovirus [2]. Unlike rotavirus, for which an effective vaccine is being used to reduce the burden of disease, there is currently no licensed vaccine nor therapeutic agent for norovirus, other than oral rehydration solution. Also, the fact that norovirus gastroenteritis incidence is high in both low- and high-income settings indicates that improvements in household water sources are 
unlikely to substantially impact disease burden [3-5]. One approach to reducing the burden of norovirus disease is to identify risk factors for infection, and then use this information to inform prevention approaches.

A known risk factor for norovirus is close contact with a symptomatic individual [6]. Transmission from food [7, 8], water [9, 10] and surfaces [11, 12] is also documented in the literature. In temperate climates, norovirus disease has been observed to peak during cold weather months and season strength is associated with average rainfall in the wettest month $[13,14]$, however, overall there is little known about environmental factors which increase the risk for norovirus disease. Information is especially sparse from low and middle income countries (LMICs).

The objective of this study is to identify environmental factors that are associated with norovirus diarrhoea in children in a LMICs setting. Using a population-based cohort of children in Leon, Nicaragua, we investigated environmental risk factors, as well as spatial and seasonal patterns of norovirus diarrhoea episodes in the community.

\section{METHODS}

The study was performed in Nicaragua's second largest city, León (2010 population: 192 164). Since the 1990s, municipal water treatment plants have been in continuous use in León. A rotavirus immunisation programme was implemented in October 2006. Studies conducted since the rotavirus vaccine introduction in León found that norovirus was the most commonly detected enteropathogen among children with diarrhoea $[15,16]$.

A simple random sample of households was selected from the Health and Demographic Surveillance Site-León (HDSS-León) [17], with children under the age of five years in each sampled household eligible for participation, as previously described [15]. The 825 participating children resided in 540 households; 206 of the children lived in the same household as another participating child.

Households were visited by female field workers who lived in the same communities as the participants. The field workers collected information on individual, family and household characteristics, and returned to the household every 14 days over a 1-year period (25 January 2010 to 24 January 2011) to assess for diarrhoea episodes during the previous 14 days. Diarrhoea was defined as an increase in stool frequency to at least three stools per 24-h period or as a substantial change in stool consistency (bloody, very loose, watery) following at least three diarrhoea-free days. Stool samples were requested for each diarrhoea episode that occurred during the study. As part of surveillance activities of the HDSS-León, GPS (Global Positioning System) receivers were used to collect geographic coordinates of each household. The geographic coordinates for the four open-air markets in León, rivers, the municipal garbage dump, and informal garbage dumps located in each city sector were also recorded.

The study was approved by the Institutional Review Boards of the Universidad Nacional Autónoma de Nicaragua, León (UNAN-León) and the University of North Carolina at Chapel Hill. Informed consent was obtained from a parent or legal guardian of each participant.

\section{Laboratory analysis}

Stool specimens were obtained in the field in a sterile plastic container or from the child's soiled diaper and then transported to the UNAN-León Microbiology Laboratory at $4{ }^{\circ} \mathrm{C}$ within $2 \mathrm{~h}$ of collection. Norovirus detection and genotyping was performed as previously described [15]. In brief, viral RNA was extracted on an automated magnetic particle processor. Real-time TaqMan RT-PCR (RT-qPCR) was performed to test for GI and GII noroviruses as described by Vega [18] with the inclusion of coliphage MS2 as extraction control. A sample was considered norovirus positive for either or both norovirus genogroup GI and GII if $C_{\mathrm{t}}$ values $\leqslant 36$. Norovirus-positive samples were genotyped after sequencing of region $\mathrm{C}$ conventional RTPCR products [19]. Genotypes were assigned using CaliciNet reference sequences [18].

As previously reported from this cohort, norovirus was the most commonly detected enteropathogen, present in $20 \%(68 / 333)$ of diarrhoeal stool samples collected and analysed; eight children experienced two norovirus diarrhoea episodes during the surveillance. Also, another enteropathogen was detected in $49 \%$ $(33 / 68)$ of diarrhoeal stools in which norovirus was detected, most commonly, Sapovirus (10/68) or Giardia lamblia (8/68) [15]. To assess asymptomatic norovirus infections in this setting, we collected stools from cohort members without diarrhoea in the previous 2 months; norovirus was detected in $13 \%$ of these stools [15].

\section{Spatial and statistical analyses}

Characteristics of interest in our analysis include age at entry into the cohort (in months), sex, if the child 
was breastfeeding at entry into the cohort, maternal education (primary or less, secondary, professional, university), maternal employment, presence of animals in the household, type of water source, type of toilet (indoor vs. latrine or none), type of walls (brick or cement vs. adobe, wood, palm, cardboard, plastic, metal), type of floor (tile, brick or cement $v s$. dirt), and distance to the nearest garbage dump, river and market. We used ArcGIS (version 10.3.1, Redlands, CA) to calculate the distance (metres) between participants' households and each environmental feature (garbage dumps, rivers and markets).

To account for potential bias due to incomplete laboratory analysis data, we created a complete case sub-cohort, which excluded any child in whom any stool sample was not collected or analysed for a reported diarrhoea episode during the surveillance period. We used $\chi^{2}$ tests and $t$ tests to compare characteristics of children with diarrhoea for whom all samples were collected and analysed to children with diarrhoea who had one or more missing samples.

Among the complete case sub-cohort we examined bivariate and multivariable associations of participant characteristics with risk of norovirus. For the bivariate analysis we used $\chi^{2}$ tests and $t$ tests to examine differences between those who experienced norovirus diarrhoea episodes and those who did not. For the multivariable models, we used Poisson models to estimate incidence rate ratios (IRR), and 95\% confidence intervals $(95 \% \mathrm{CI})$. GEE (Generalized estimating equations) with an exchangeable correlation matrix were used to account for clustering of more than one child within a household. Person time was calculated as days under surveillance excluding days sick with norovirus diarrhoea. Each individual, family and environmental factor was modelled separately and adjusted for sex and age. For these models we standardised the distance measures by subtracting the mean and dividing by the standard deviation. We conducted a sensitivity analysis to explore how our results would compare with two extreme scenarios for which there were no missing laboratory results. In the first scenario, all diarrhoea episodes with missing laboratory analysis data were assumed to be negative for norovirus ('negative scenario'), and in the second scenario, all missing laboratory analysis data were assumed to be positive for norovirus ('positive scenario'). We used SAS (version 9.3.1, Cary, NC) for statistical analyses.

To describe patterns of norovirus diarrhoea episodes within households of study participants, we identified episodes of the same genotype detected in a 30-day time window within the same household for the 206 children who shared a household with another participating child. We also identified norovirus diarrhoea episodes of different genotypes within a 30-day time window in households. To describe patterns of norovirus diarrhoea episodes in nearby households, we used ArcGIS to create $500 \mathrm{~m}$ buffers around the household of each case detected during the study. We then identified episodes of the same genotype detected in a 30-day time window within each buffer. Because this was a descriptive analysis, we included all observed norovirus episodes from the entire cohort.

To describe seasonal patterns of norovirus diarrhoea episodes, we reported on norovirus genotypes detected in samples collected by quarter of the year: January-March (Quarter 1), April-June (Quarter 2), July-September (Quarter 3), and October-December (Quarter 4). In León, Quarter 1 is the peak of the dry season, Quarter 2 is the beginning of the rainy season, Quarter 3 is the peak of the rainy season, and Quarter 4 is the beginning of the dry season.

\section{RESULTS}

In the study, stool samples were collected and analysed for 333 of the 677 reported episodes of diarrhoea $(49 \%)$. These data were missed due to (1) caregiver did not provide a sample, (2) a sample was collected, but it was not received by the UNAN Microbiology Laboratory within a 2-h period according to the study protocol, or (3) a sample was captured, but norovirus laboratory analysis was not performed due to insufficient quantity. The overall cohort $(N=825)$ had similar characteristics as the complete case subcohort $(N=588)$, which did not have any missing laboratory analysis data, as shown in Table 1. Characteristics were similar in children with diarrhoea with complete stool analysis data as compared with children with diarrhoea with any missing stool analysis data, except that those with complete stool analysis data were more likely to have a non-dirt floor $(88 \% v s .71 \%$, $P=0.0004)$ and brick or cement walls (91\% vs. $83 \%, P$ $=0.05)$ (Supplementary Table S1). In the complete case sub-cohort, we observed 38 norovirus episodes with six children experiencing norovirus twice and 26 children had norovirus once during study observation.

Norovirus diarrhoea was more commonly experienced by younger children $(P<0 \cdot 001)$ and those with animals in their household $(P=0.02)$ in the complete case sub-cohort (Table 2). On additional investigation, there was not a significant association between 
Table 1. Individual, family and environmental characteristics of children in the overall cohort $(n=825)$ and those included in the complete case sub-cohort $(n=588)$

\begin{tabular}{|c|c|c|c|c|}
\hline & \multicolumn{2}{|c|}{ Overall $(n=825)$} & \multicolumn{2}{|c|}{ Complete case $(n=588)$} \\
\hline & $\%(n)$ & mean (s.D.*) & $\%(n)$ & mean (s.D.) \\
\hline \multicolumn{5}{|l|}{ Individual } \\
\hline Female & $50 \%(409)$ & & $49 \%(285)$ & \\
\hline Age, months & & $29(17)$ & & $30(17)$ \\
\hline Breastfed at study entry $^{\dagger}$ & $77 \%(127)$ & & $79 \%(88)$ & \\
\hline \multicolumn{5}{|l|}{ Family } \\
\hline \multicolumn{5}{|l|}{ Maternal education } \\
\hline Primary or less & $23 \%(186)$ & & $23 \%(136)$ & \\
\hline Secondary & $50 \%(408)$ & & $47 \%(279)$ & \\
\hline Professional & $12 \%(99)$ & & $13 \%(75)$ & \\
\hline University & $16 \%(132)$ & & $17 \%(98)$ & \\
\hline Mother employed & $40 \%(329)$ & & $40 \%(237)$ & \\
\hline \multicolumn{5}{|l|}{ Environmental } \\
\hline Animals in household & $62 \%(508)$ & & $59 \%(344)$ & \\
\hline Indoor municipal water source & $97 \%(800)$ & & $98 \%(574)$ & \\
\hline Indoor toilet $\S^{\S}$ & $80 \%(661)$ & & $80 \%(470)$ & \\
\hline Brick or cement walls & $88 \%(722)$ & & $89 \%(526)$ & \\
\hline Non-dirt floor** & $77 \%(638)$ & & $80 \%(469)$ & \\
\hline Distance to closest dump, metres & & $726(307)$ & & $732(308)$ \\
\hline Distance to closest river, metres & & $667(411)$ & & $644(406)$ \\
\hline Distance to closest market, metres & & $929(391)$ & & $922(394)$ \\
\hline
\end{tabular}

* s.D., standard deviation.

$\dagger$ For those $\leqslant 12$ months; $n=165$ in the overall cohort and $n=112$ for the complete case sub-cohort.

$\$$ Other water sources include: community municipal well, well, buy water.

$\S$ Other types of sanitation include: latrine, no toilet.

ๆ Other types of walls include: adobe, wood, palm, cardboard, plastic, metal.

** Tile, brick, or cement floor.

the presence of an individual type of animal examined (dogs, cats, chickens, pigs or cattle) and experiencing a norovirus diarrhoea episode. Further, in these bivariate analyses, having an indoor toilet and having a non-dirt floor tended to be associated with norovirus diarrhoea, however, these associations did not reach statistical significance $(P$-values $=0.12$ for both associations).

In the adjusted multivariable models, younger age was strongly associated with an increased risk of norovirus diarrhoea, but significant associations were not observed for the environmental factors examined (Table 3). Children with more educated mothers tended to be at increased risk of norovirus diarrhoea. A sensitivity analysis showing two extreme scenarios, where all missing stool analysis data were either considered to be negative $v s$. considered to be positive is shown in Supplementary Table S2. In these analyses, age is consistently associated with norovirus diarrhoea across models. Further, while many of the estimates remain similar or move closer to the null, in the negative scenario, the association between indoor toilet and norovirus diarrhoea becomes stronger.

In the entire cohort we observed 68 norovirus episodes and further examination illustrates matched genotypes in cohort members living in the same household or in different households in close proximity. There were five cases in which norovirus diarrhoea episodes of the same genotype were detected in households with more than one study participant (Table 4); these noroviruses were both GI and GII. In a sixth case, two children in the same household became symptomatic on the same day with a norovirus diarrhoea episode; however, genotyping results were not definitive in one of the two children. In comparison, there were two norovirus diarrhoea episodes due to non-matched genotypes shared between household members during any 30 day time window in the surveillance period (data not shown). There were nine cases in which norovirus diarrhoea episodes of the same genotype were detected within $500 \mathrm{~m}$ buffers (Table 5). All but one of these matched episodes 
Table 2. Individual, family and environmental characteristics of children who did vs. did not have a Norovirus diarrhoea episode detected during one year of surveillance in the complete case sub-cohort $(n=588)$

\begin{tabular}{|c|c|c|c|c|c|}
\hline & \multicolumn{2}{|c|}{$\begin{array}{l}\text { No norovirus episode } \\
\text { detected }(N=556)\end{array}$} & \multicolumn{2}{|c|}{$\begin{array}{l}\text { Norovirus episode } \\
\text { detected }(N=32 *)\end{array}$} & \multirow{2}{*}{$P$-value for difference } \\
\hline & $\%(n)$ & Mean (S.D. ${ }^{\dagger}$ ) & $\%(n)$ & Mean (S.D.) & \\
\hline \multicolumn{6}{|l|}{ Individual } \\
\hline Sex $(\%$ female $)$ & $49 \%(272)$ & & $41 \%(13)$ & & $0 \cdot 36$ \\
\hline Age, months & & $31(17)$ & & $18(14)$ & $<0 \cdot 0001$ \\
\hline Breastfed at study entry & $79 \%(77)$ & & $75 \%(12)$ & & $0 \cdot 75$ \\
\hline \multicolumn{6}{|l|}{ Family } \\
\hline \multicolumn{6}{|l|}{ Maternal education } \\
\hline Primary or less & $23 \%(136)$ & & $19 \%(6)$ & & $0 \cdot 16$ \\
\hline Secondary & $48 \%(268)$ & & $34 \%(11)$ & & \\
\hline Professional & $12 \%(68)$ & & $22 \%(7)$ & & \\
\hline University & $16 \%(90)$ & & $25 \%(8)$ & & \\
\hline Mother employed & $41 \%(225)$ & & $38 \%(12)$ & & $0 \cdot 74$ \\
\hline \multicolumn{6}{|l|}{ Environmental } \\
\hline Animals in household & $57 \%(319)$ & & $78 \%(25)$ & & $0 \cdot 02$ \\
\hline Indoor municipal water source ${ }^{\S}$ & $98 \%(542)$ & & $100 \%(32)$ & & $1 \cdot 00$ \\
\hline Indoor toilet & $79 \%(441)$ & & $91 \%(29)$ & & $0 \cdot 12$ \\
\hline Brick or cement walls** & $90 \%(498)$ & & $88 \%(28)$ & & $0 \cdot 76$ \\
\hline Non-dirt floor ${ }^{\dagger \dagger}$ & $79 \%(440)$ & & $91 \%(29)$ & & $0 \cdot 12$ \\
\hline Distance to closest garbage dump, metres & & 735 (309) & & $693(279)$ & $0 \cdot 46$ \\
\hline Distance to closest river, metres & & $642(404)$ & & $684(441)$ & $0 \cdot 57$ \\
\hline Distance to closest market, metres & & $922(394)$ & & $914(394)$ & $0 \cdot 91$ \\
\hline
\end{tabular}

* 32 children experienced a total of 38 total norovirus diarrhoea episodes.

$\dagger$ S.D., standard deviation.

$\$$ For those $\leqslant 12$ months at study entry; $n=112$.

$\S$ Other water sources include: community municipal well, well, buy water.

I Other types of sanitation include: latrine, no toilet.

** Other types of walls include: adobe, wood, palm, cardboard, plastic, metal.

$\dagger \dagger$ Tile, brick or cement floor.

within buffers were GII, and the majority were GII.4, as compared with the genotype distribution in the overall cohort (Fig. 1). In summary, of the 68 norovirus episodes we observed, five episodes had matching genotypes to an episode experienced by a cohort member within the same household, and nine episodes had matching genotypes to an episode experienced by a cohort member within the same buffer zone.

Norovirus diarrhoea episodes peaked during AprilJune of the study year, which corresponded to the early rainy season in León. The distribution of norovirus genotypes by quarter of the year is shown in Figure 1. Co-infections with GI and GII genotypes were only found during the rainy season months.

\section{DISCUSSION}

We examined associations between norovirus diarrhoea episodes and individual, family and environmental factors among children in a cohort study. In the cohort of children analysed, norovirus diarrhoea was more commonly experienced among younger children and those with animals in their households, and tended to be more common among those with an indoor toilet, and those with non-dirt floors. However, multivariable analyses showed only modest associations between these environmental factors and risk of norovirus diarrhoea. Interestingly, norovirus diarrhoea risk was not associated with low socio-economic status, and actually tended to be more common among children of mothers with post-secondary education as compared with mothers with less education in multivariable models. Although data from other LMICs are sparse, this finding may be in contrast to a study conducted in England, which found that children of lower socioeconomic status had a higher odds of norovirus gastroenteritis [20]. However, another study conducted in Netherlands did not find differences in detection of 
Table 3. Individual, family and environmental factors associated with Norovirus diarrhoea episodes in multivariable analysis

\begin{tabular}{|c|c|c|}
\hline & $\begin{array}{l}\text { Unadjusted models } \\
n=588 \\
\text { IRR }(95 \% \mathrm{CI})\end{array}$ & $\begin{array}{l}\text { Adjusted models* } \\
n=588 \\
\text { IRR }(95 \% \mathrm{CI})\end{array}$ \\
\hline \multicolumn{3}{|l|}{ Individual } \\
\hline Female & Ref. & Ref. \\
\hline Male & $1 \cdot 42(0 \cdot 70-2 \cdot 89)$ & $1 \cdot 69(0 \cdot 82-3 \cdot 48)$ \\
\hline Age, months & $0.95(0.93-0.97)$ & $0.95(0.93-0.97)$ \\
\hline Breastfed $^{\dagger}$ & Ref. & Ref. \\
\hline Not breastfed & $0 \cdot 71(0 \cdot 20-2 \cdot 52)$ & $0 \cdot 63(0 \cdot 15-2 \cdot 62)$ \\
\hline \multicolumn{3}{|l|}{ Family } \\
\hline \multicolumn{3}{|l|}{ Maternal education } \\
\hline Secondary & Ref. & Ref. \\
\hline Primary or less & $1 \cdot 23(0 \cdot 46-3 \cdot 34)$ & $1 \cdot 40(0 \cdot 53-3 \cdot 70)$ \\
\hline Professional & $2 \cdot 20(0 \cdot 82-5 \cdot 94)$ & $2 \cdot 46(0 \cdot 93-6 \cdot 55)$ \\
\hline University & $2 \cdot 34(0 \cdot 93-5 \cdot 84)$ & $2 \cdot 41(0 \cdot 97-6 \cdot 02)$ \\
\hline Mother employed & Ref. & Ref. \\
\hline Mother not employed & $1 \cdot 54(0 \cdot 73-3 \cdot 23)$ & $1 \cdot 12(0 \cdot 54-2 \cdot 34)$ \\
\hline \multicolumn{3}{|l|}{ Environmental } \\
\hline No animals in home & Ref. & Ref. \\
\hline Animals in home & $2 \cdot 08(0 \cdot 83-5 \cdot 22)$ & $1 \cdot 77(0 \cdot 71-4 \cdot 42)$ \\
\hline Indoor toilet & Ref. & Ref. \\
\hline Other sanitation & $0 \cdot 36(0 \cdot 11-1 \cdot 15)$ & $0 \cdot 39(0 \cdot 12-1 \cdot 27)$ \\
\hline Brick or cement walls & Ref. & Ref. \\
\hline Other walls ${ }^{\S}$ & $1.55(0.53-4.52)$ & $1.49(0 \cdot 55-4.08)$ \\
\hline Non-dirt floor & Ref. & Ref. \\
\hline Dirt floor & $0 \cdot 39(0 \cdot 12-1 \cdot 25)$ & $0 \cdot 42(0 \cdot 14-1 \cdot 31)$ \\
\hline Distance to closest garbage dump** & $1 \cdot 21(0 \cdot 91-1 \cdot 61)$ & $1 \cdot 20(0 \cdot 89-1 \cdot 63)$ \\
\hline Distance to closest river ${ }^{\dagger \dagger}$ & $1.29(0.88-1.89)$ & $1.28(0.85-1.92)$ \\
\hline Distance to closest market & $1.27(0.88-1.83)$ & $1.27(0 \cdot 86-1 \cdot 86)$ \\
\hline
\end{tabular}

* Each characteristic is adjusted for age and gender, except the model with age is adjusted only for gender and the model for gender is only adjusted for age.

$\dagger$ Breastfed at study entry for those $\leqslant 12$ months, $N=112$.

$\$$ Other types of sanitation include: latrine, no toilet.

$\S$ Other types of walls include: adobe, wood, palm, cardboard, plastic, metal.

I Tile, brick or cement floor.

** Standard deviation for distance to garbage dump is $307 \mathrm{~m}$.

$\dagger$ Standard deviation for distance to river is $411 \mathrm{~m}$.

\# Standard deviation for distance to market is $391 \mathrm{~m}$.

norovirus in stools of children attending daycare centers by socio-economic status [21]. More data are needed to understand whether there is an association between socio-economic status and risk of norovirus, and whether this relationship varies in high- vs. lowincome countries.

While norovirus has not been proven to be a zoonotic pathogen [22], in our analysed cohort, norovirus diarrhoea was more common among children with an animal present in the home. A study of children in the Netherlands found an association between exposure to livestock and detection of norovirus in stool [23]. Finally, although the association in our analysed cohort was not significant, we were surprised to find that having an indoor toilet tended to be associated with norovirus diarrhoea. Prior studies have reported on the association between norovirus episodes and contaminated toilets on cruise ships [24], and norovirus has been identified on toilet surfaces $[25,26]$. Therefore, it is conceivable that if an indoor toilet is contaminated with infectious vomitus or stool from a household member with norovirus infection, a child in the household is more likely to have contact with the infectious material than if it were in an outdoor latrine. Future study is warranted to further investigate this exploratory association. 
Table 4. Norovirus diarrhoea episodes of the same genotype in the same household

\begin{tabular}{lllll}
\hline \hline Household code & Child & Age (in months) & $\begin{array}{l}\text { Days between } \\
\text { symptom onset }\end{array}$ & Genotype \\
\hline A & 1 & 22 & & GI.3B \\
A & 2 & 7 & 7 & GI.3B \\
B & 1 & 28 & 1 & GI.2 \\
B & 2 & 5 & 5 & GI.2 \\
C & 1 & 1 & $5 I .13$ \\
C & 2 & 29 & 4 & GII.13 \\
D & 1 & 15 & & GII.6 \\
D & 2 & 13 & 24 & GII.6 \\
E & 1 & 25 & & GII.4 New Orleans \\
E & 2 & 11 & 0 & GI.3B and GII.8 \\
F & 1 & 13 & Could not identify* \\
F & 2 & 13 & \\
\hline \hline
\end{tabular}

* Positive by norovirus qPCR screening, but genotyping results not definitive.

Table 5. Norovirus diarrhoea episodes of the same genotype in different households within a $500 \mathrm{~m}$ buffer

\begin{tabular}{lllll}
\hline \hline Buffer zone & Child & Age (in months) & $\begin{array}{l}\text { Days between } \\
\text { symptom onset }\end{array}$ & Genotype \\
\hline A & 1 & 6 & & GII.4_New Orleans \\
A & 2 & 11 & 5 & GII.4_New Orleans \\
B & 1 & 36 & 2 & GII.4_Den Haag \\
B & $2^{*}$ & 7 & & GII.4_Den Haag \\
C & 1 & 25 & 2 & GII.4_New Orleans \\
C & $2^{\dagger}$ & 15 & & GII.4_New Orleans \\
D & 1 & 2 & 14 & GII.14 \\
D & 2 & 22 & 3 & GII.14 \\
E & 1 & 26 & 5 & GII.4_New Orleans \\
E & 2 & 25 & & GII.4_New Orleans \\
E & $3^{\dagger}$ & 15 & 3 & GII.4_Den Haag \\
F & 1 & 18 & 5 & GII.4_Den Haag \\
F & 2 & 36 & & GII.4_Den Haag \\
F & $3^{*}$ & 7 & 12 & GII.14 \\
G & 1 & 14 & & GII.14 \\
G & 2 & 47 & 21 & GII.4_Den Haag \\
H & 1 & 9 & & GII.4_Den Haag \\
H & $2^{*}$ & 16 & 27 & GII.4_Den Haag and GI.3B \\
I & 1 & 10 & & GII.4_Den Haag \\
I & $2^{*}$ & 16 & 17 & GI.3B \\
J & 1 & 40 & 29 & GI.3B and GII.14 \\
J & 2 & & & \\
\hline
\end{tabular}

* Denotes that child could be a secondary case in zone B or F.

$\dagger$ Denotes that child could be a secondary case in zone $\mathrm{C}$ or $\mathrm{E}$.

$\$$ Denotes that child could be a secondary case in zone $\mathrm{H}$ or $\mathrm{I}$.

Further, we identified norovirus diarrhoea episodes of the same genotype within households and in $500 \mathrm{~m}$ buffer zones around households of children with norovirus diarrhoea episodes. Interestingly, while GI infections were detected among two participants in the same household, among participants with norovirus in the same buffer zone, almost all infections were GII noroviruses. This may reflect the high infectivity of GII noroviruses [27], and potentially, the requirement for more intense or repeated contact for transmission of GI noroviruses.

A limitation of our study is that upon exclusion of children with incomplete stool analysis data, the 


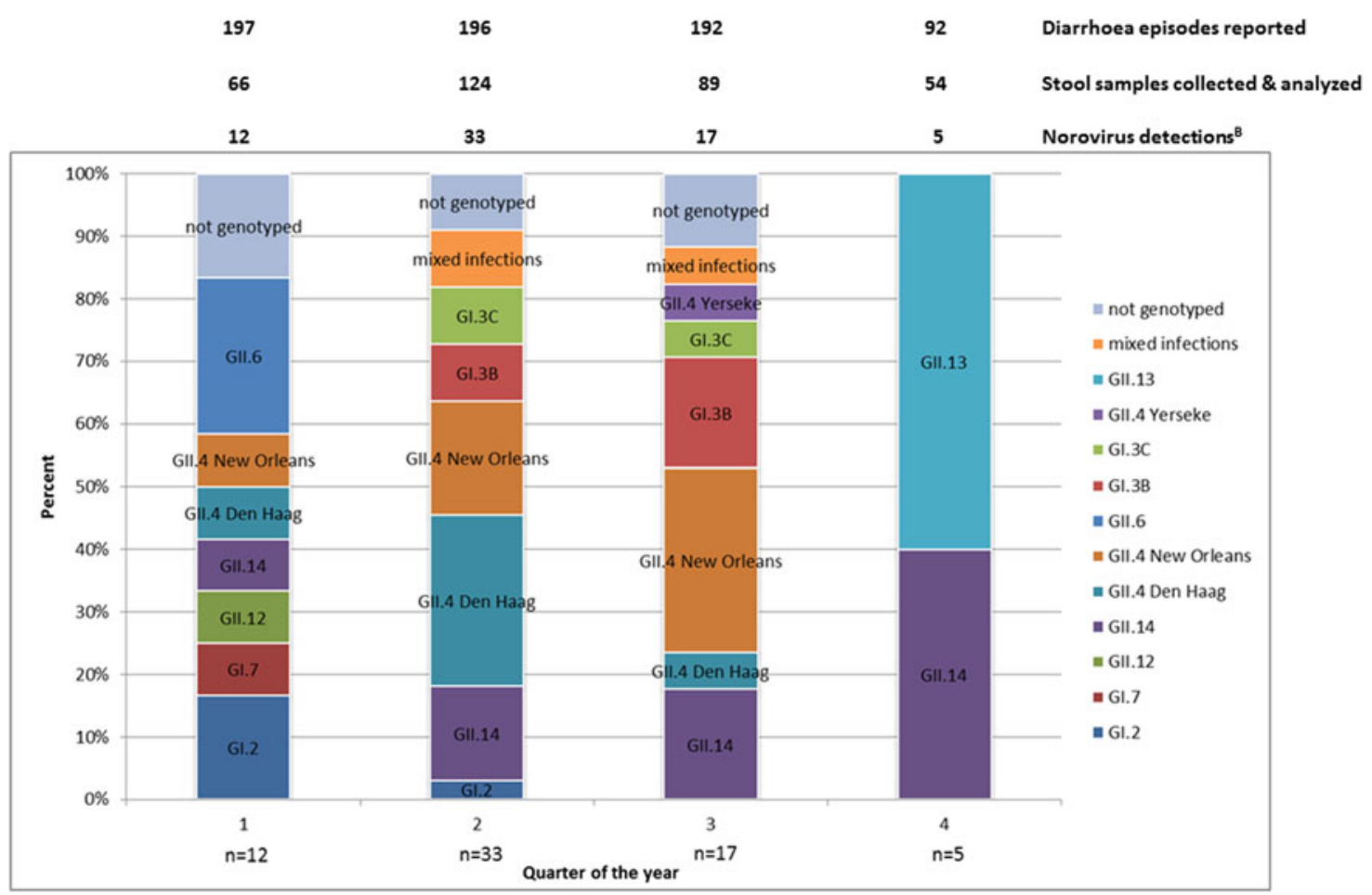

Fig. 1. Norovirus genotypes identified in diarrhoea samples by quarter ${ }^{\mathrm{A}}$ of the year. ${ }^{\mathrm{A}}$ Quarter $1=\mathrm{January}-\mathrm{March}$; Quarter 2=April-June; Quarter 3= July-September; Quarter 4= October-December. ${ }^{\mathrm{B}}$ Date of episode missing for one stool sample.

numbers of children with norovirus diarrhoea were not large enough to support inclusion of more potential confounders in multivariable models. Another limitation of the study is that co-infections with other enteric pathogens were common, and therefore, it cannot be determined whether the diarrhoea symptoms were caused by norovirus, by the other enteropathogen or by a combination of the two enteropathogens. Finally, not all members of the community were included in the study, as only a random sample of children under age five years living in the HDSS were followed. Therefore, we cannot confirm that participants with the same genotype within households or buffer zones transmitted the virus, or whether other individuals not under surveillance were responsible for the norovirus diarrhoea episode. Further, in our evaluation of proximity to environmental features, it is acknowledged that other features may warrant evaluation. For example, while we evaluated the risk associated with living near rivers, we did not assess the risk of living close to smaller or intermittent tributaries of these rivers, which were not included in our spatial database.

In conclusion, in this paediatric cohort study, environmental factors were only modestly associated with risk of norovirus diarrhoea, however factors such as the presence of animals in the household, indoor toilets, and dirt floors warrant investigation in future studies. Both GI and GII noroviruses of the same genotype were detected among children with diarrhoea living in the same household, however, primarily GII genotypes were shared between children with diarrhoea living in different households in close proximity. Finally, in this tropical setting, norovirus diarrhoea peaked during the early rainy season.

\section{SUPPLEMENTARY MATERIAL}

The supplementary material for this article can be found at https://doi.org/10.1017/S0950268817000322

\section{ACKNOWLEDGEMENTS}

This study received funding from the Merck Investigator-Initiated Studies Program (S.B-D., 36822, laboratory analysis) and the Thrasher Research Fund (S.B-D., NR-0072, field work). S.B-D. was supported by the Fogarty International Center at the National Institutes of Health (5K01TW008401-04). F.B. received 
support for calicivirus research in Nicaragua from NETROPICA (05-N-2010).

\section{DECLARATION OF INTEREST}

F.E. and M.P. have received research funding from Takeda Pharmaceutical Company Ltd.

\section{ETHICAL STANDARDS}

The authors assert that all procedures contributing to this work comply with the ethical standards of the relevant national and institutional committees on human experimentation and with the Helsinki Declaration of 1975, as revised in 2008

\section{REFERENCES}

1. Fischer Walker CL, et al. Diarrhoea incidence in lowand middle-income countries in 1990 and 2010: a systematic review. BMC Public Health 2012; 12: 220.

2. Ahmed SM, et al. Global prevalence of norovirus in cases of gastroenteritis: a systematic review and meta-analysis. Lancet Infectious Diseases 2014; 14: 725-730.

3. Shioda K, et al. Population-based incidence rates of diarrhoeal disease associated with Norovirus, Sapovirus, and astrovirus in Kenya. PLoS ONE 2016; 11: e0145943.

4. Grytdal SP, et al. Incidence of Norovirus and other viral pathogens that cause acute gastroenteritis (AGE) among Kaiser Permanente member populations in the United States, 2012-2013. PLoS ONE 2016; 11: e0148395.

5. O'Brien SJ, et al. Age-specific incidence rates for Norovirus in the community and presenting to primary healthcare facilities in the United Kingdom. Journal of Infectious Diseases 2016; 213: S15-S18.

6. Fretz R, et al. Risk factors for infections with Norovirus gastrointestinal illness in Switzerland. European Journal of Clinical Microbiology \& Infectious Diseases 2005; 24: 256-261.

7. Daniels NA, et al. A foodborne outbreak of gastroenteritis associated with Norwalk-like viruses: first molecular traceback to deli sandwiches contaminated during preparation. Journal of Infectious Diseases 2000; 181: 1467-1470.

8. Franck KT, et al. Calicivirus contamination in foodborne outbreaks in Denmark, 2005-2011--the role of the asymptomatic food handler. Journal of Infectious Diseases 2015; 211: 563-570.

9. Kukkula M, et al. Outbreak of viral gastroenteritis due to drinking water contaminated by Norwalk-like viruses. Journal of Infectious Diseases 1999; 180: 1771-1776.

10. Anderson AD, et al. A waterborne outbreak of Norwalk-like virus among snowmobilers-Wyoming, 2001. Journal of Infectious Diseases 2003; 187: 303-306.

11. Cheesbrough JS, et al. Widespread environmental contamination with Norwalk-like viruses (NLV) detected in a prolonged hotel outbreak of gastroenteritis. Epidemiology and Infection 2000; 125: 93-98.

12. Hall AJ, et al. Updated norovirus outbreak management and disease prevention guidelines. $M M W R$ Recommendations and Reports 2011; 60: 1-18.

13. Ahmed SM, et al. A systematic review and meta-analysis of the global seasonality of norovirus. PLOS ONE 2013; 8: e75922.

14. Mounts AW, et al. Cold weather seasonality of gastroenteritis associated with Norwalk-like viruses. Journal of Infectious Diseases 2000; 181: S284-S287.

15. Becker-Dreps $\mathbf{S}$, et al. Etiology of childhood diarrhoea after rotavirus vaccine introduction: a prospective, population-based study in Nicaragua. Pediatric Infectious Disease Journal 2014; 33: 1156-1163.

16. Bucardo F, et al. Predominance of Norovirus and Sapovirus in Nicaragua after implementation of universal rotavirus vaccination. PLoS ONE 2014; 9: e98201.

17. Pena R, et al. The Nicaraguan Health and Demographic Surveillance Site, HDSS-Leon: a platform for public health research. Scandinavian Journal of Public Health 2008; 36: 318-325.

18. Vega E, et al. Novel surveillance network for norovirus gastroenteritis outbreaks, United States. Emerging Infectious Diseases 2011; 17: 1389-1395.

19. Kojima S, et al. Genogroup-specific PCR primers for detection of Norwalk-like viruses. Journal of Virological Methods 2002; 100: 107-114.

20. Phillips G, et al. Risk factors for symptomatic and asymptomatic norovirus infection in the community. Epidemiology and Infection 2011; 139: 1676-1686.

21. Pijnacker R, et al. Characteristics of child daycare centres associated with clustering of major enteropathogens. Epidemiology and Infection 2016; 144: 2527-2539.

22. Karst SM, et al. What is the reservoir of emergent human norovirus strains? Journal of Virology 2015; 89: 5756-5759.

23. Heusinkveld M, et al. Potential causative agents of acute gastroenteritis in households with preschool children: prevalence, risk factors, clinical relevance and household transmission. European Journal of Clinical Microbiology \& Infectious Diseases 2016; 35: 1691-1700.

24. Chimonas MA, et al. Passenger behaviors associated with norovirus infection on board a cruise ship--Alaska, May to June 2004. Journal of Travel Medicine 2008; 15: 177-183.

25. Jones EL, et al. Role of fomite contamination during an outbreak of norovirus on houseboats. International Journal of Environmental Health Research 2007; 17: 123-131.

26. Gallimore CI, et al. Environmental monitoring for gastroenteric viruses in a pediatric primary immunodeficiency unit. Journal of Clinical Microbiology 2006; 44: 395-399.

27. Matthews JE, et al. The epidemiology of published norovirus outbreaks: a review of risk factors associated with attack rate and genogroup. Epidemiology and Infection 2012; 140: 1161-1172. 